FRIDAY, 14 JUNE 2019

\section{Reproductive issues in rheumatology}

\section{OP0326 DEVELOPMENT OF A STANDARDIZED MINIMAL CORE DATA SET FOR PREGNANCY REGISTERS IN RHEUMATOLOGY - RESULTS OF A EULAR TASK FORCE}

Yvette Meißner ${ }^{1}$, Rebecca Fischer-Betz ${ }^{2}$, Laura Andreoli ${ }^{3}$, Nathalie CostedoatChalumeau $^{4,5}$, Diederik De Cock ${ }^{6}$, Radboud Dolhain ${ }^{7}$, Frauke Förger ${ }^{8,9}$, Doreen Goll ${ }^{10}$, Anna Moltót 5,11 , Rebecca Özdemir ${ }^{12}$, Luigi Raio ${ }^{8,9}$, Catherine Nelson-Piercy ${ }^{13}$, Sebastian C Rodriguez-García ${ }^{14}$, Savino Sciascia ${ }^{15}$ Marianne Wallenius ${ }^{16}$, Astrid Zbinden ${ }^{8}$, Angela Zink ${ }^{1,17}$, Anja Strangfeld ${ }^{1}{ }^{1}$ German Rheumatism Research Centre, Berlin, Germany, ${ }^{2}$ Heinrich-Heine University and Hiller Research Unit, Düsseldorf, Germany, ${ }^{3}$ University of Brescia, Brescia, Italy; ${ }^{4}$ Université Paris-Descartes, Paris, France; ${ }^{5} \mathrm{Hopital}$ Cochin, Paris, France; ${ }^{6} \mathrm{KU}$ Leuven, Leuven, Belgium; ' ${ }^{7}$ rasmus University Medical Center, Rotterdam, Netherlands; ${ }^{8}$ University Hospital, Bern, Switzerland; ${ }^{9}$ University of Bern, Bern, Switzerland; ${ }^{10}$ Patient partner, Berlin, Germany; ${ }^{11}$ INSERM Unit 1153, CRESS, Paris, France; ${ }^{12}$ Patient partner, Duisburg, Germany; ${ }^{13}$ St. Thomas Hospital, London, United Kingdom; ${ }^{14}$ Hospital Clínic Barcelona, Barcelona, Spain; ${ }^{15}$ University di Torino, Torino, Italy; ${ }^{16}$ University Hospital and Norwegian University of Science and Technology, Trondheim, Norway, ${ }^{17}$ Charité University Medicine, Berlin, Germany

Background: Results from individual data collections on drug safety during preg nancy and outcomes of pregnancy in patients with inflammatory rheumatic diseases (IRD) are often limited due to small number of cases. Joint data analyses from different data sources could solve this problem.

Objectives: The aim of this EULAR task force was to define a core data set to facilitate joint analysis of pregnancy registers in rheumatology.

Methods: Scope and core areas of the core data set have been developed according to COS-STAD recommendations ${ }^{1}$ by consensus. An initial list of data items possibly relevant for pregnancy registers was generated based on (I) a systematic literature search, (II) data items already collected by European pregnancy registers and (III) a survey amongst patient representatives. Consensus about the importance of each data item to be included in a core data set was reached by applying a 2-round Delphi survey where each item was rated on a numeric scale (1-3 = low importance, 4-6 = important but not critical, 7-9 = critical importance). Any data item that was considered as 'critical important' by at least $70 \%$ of responders in Delphi round 2 was included in the core set. During a face-to-face meeting of the EULAR task force, the inclusion or exclusion of data items with no consensus was finally decided.

Results: The scope was defined as follows: 'To develop a standardized core data set for data collection in prospective observational research and clinical care of pregnant women with IRD including the neonatal phase. All interventions the women receive will be covered. Patients should be enrolled at the earliest possible moment during pregnancy, and data should ideally be collected once every trimester. An additional visit should capture the neonatal phase'. Three core areas were described: 'Maternal information' (including demographics, IRD disease characteristics and comorbidities), 'Pregnancy' (including prior and current pregnancy(ies), delivery and neonatal outcomes) and 'Treatment' (including medication of IRD and other health conditions).

64 experts from 14 different European countries participated in the 2 rounds of Delphi (68\% female; $84 \%$ physicians, $5 \%$ obstetricians, $5 \%$ epidemiologists, $3 \%$ patients, $3 \%$ midwives). Of the 148 data items, 85 were included in the final core set. The figure shows included items within the core areas.

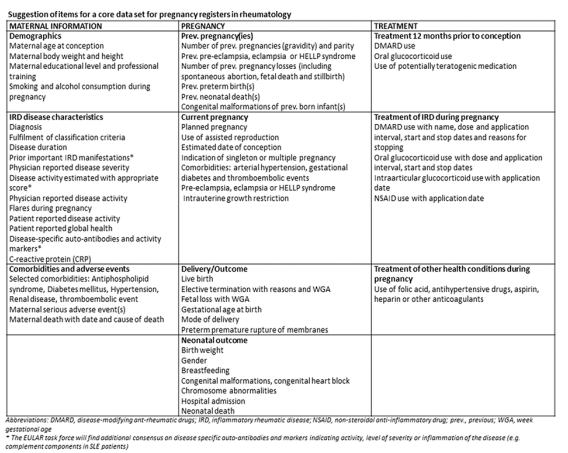

Conclusion: The consensus process resulted in an extensive list of data items recommended by experts to be collected as a minimum by pregnancy registers in rheumatology. This core data set applies to all pregnant women irrespective of the underlying IRD. The EULAR task force plans to find consensus on additional disease specific advice.

\section{REFERENCE:}

[1] Kirkham et. al. PLoS med.2017;14(11):e1002447.

Acknowledgement: This work was supported by a research grant from FOREUM Foundation for Research in Rheumatology.

Disclosure of Interests: Yvette Meißner Speakers bureau: Pfizer, Rebecca Fischer-Betz Grant/research support from: GlaxoSmithKline and UCB Pharma for performing the LuLa-study., Laura Andreoli: None declared, Nathalie CostedoatChalumeau: None declared, Diederik De Cock: None declared, Radboud Dolhain Grant/research support from: UCB Pharma B.V, Frauke Förger: None declared, Doreen Goll: None declared, Anna Moltó: None declared, Rebecca Özdemir: None declared, Luigi Raio: None declared, Catherine Nelson-Piercy Consultant for: UCB, Speakers bureau: UCB, Sebastian C Rodriguez-García: None declared, Savino Sciascia: None declared, Marianne Wallenius: None declared, Astrid Zbinden: None declared, Angela Zink Speakers bureau: Speakers fees from AbbVie, Janssen, Pfizer, Roche, Sanofi, Anja Strangfeld Speakers bureau: Speakers fees from Bristol-Myers Squibb, MSD, Pfizer, Roche

DOI: 10.1136/annrheumdis-2019-eular.402

\section{FRIDAY, 14 JUNE 2019}

\section{The future of therapeutic strategies}

\section{OP0327 FECAL MICROBIOTA TRANSPLANTATION IN SYSTEMIC SCLEROSIS: A DOUBLE-BLIND, PLACEBO- CONTROLLED RANDOMIZED PILOT TRIAL}

Anna-Maria Hoffmann-Vold, Håvard Fretheim, Brian K Chung, Henriette Didriksen, Espen S Bækkevold, Øyvind Midtvedt, Cathrine Brunborg, Torhild Garen,

Tore Midtvedt, Johannes R Hov, Knut Ea Lundin, Øyvind Molberg. Oslo University Hospital, Oslo, Norway

Background: Systemic sclerosis (SSc) is a progressive, multi organ, autoimmune disease marked by frequent and severe gastrointestinal (Gl) afflictions and gut dysbiosis.

Objectives: Determine the safety and efficacy of fecal microbiota transplantation (FMT) using commercially-available anaerobic cultivated human intestinal microbiota (ACHIM) in patients with SSc.

Methods: The trial was a single-center, randomized, double-blind, placebo-controlled 16-week pilot of FMT by gastroduodenoscopy of ACHIM in SSc conducted at Oslo University Hospital. Primary endpoints were safety and clinical efficacy on Gl symptoms assessed at weeks 4 and 16 . Safety was assessed by observation, interviews and standardized safety form. Efficacy on Gl symptoms was measured using the UCLA GIT 2.0 score questionnaire. Patients were defined as responders if reported symptom improvement was equivalent to the UCLA GIT definition of "minimally clinically important difference". Secondary and explorative endpoints included changes in relative abundance of total, immunoglobulin (Ig)A- and IgMcoated fecal bacteria measured by 16s rRNA sequencing; changes in modified Rodnan skin score, lung function, CRP, ESR, and patient and physician global Descriptive statistics were applied for clinical endpoints and linear mixed models for microbial analysis.

Results: Ten patients with limited cutaneous SSc randomized to ACHIM ( $n=5)$ or placebo $(n=5)$ were included. All patients were female with clinical apparent $G$ symptoms, mean age of 62 years and mean time from diagnosis of $12 \mathrm{yrs}$. Two placebo controls experienced procedure-related serious adverse events; one developed laryngospasms at first gastroduodenoscopy necessitating study exclusion, one duodenal perforation at final gastroduodenoscopy. Improvement in total GIT score was reported by 3/5 FMT patients compared to 2/4 placebo controls at weeks 4 and 16 (Figure 1). FMT effects were most pronounced on lower Gl symptoms, with improvement reported by 5/5 FMT patients with diarrhea, distention/ bloating and/or fecal incontinence at baseline compared to 2/4 placebo controls (Figure). Clinical secondary endpoints showed no differences and other side effects (stomach discomfort, bloating and diarrhea) were mild and transient. Fecal microbiota diversity (observed number of operational taxonomic units) was increased after FMT compared to placebo treatment at week $16(p<0.006)$. Moreover, abundant bacterial genera in ACHIM were present within the total, and IgAand IgM-coated fecal bacteria at both week 4 and 16 in the FMT group (Figure 2) but not in the placebo controls 
Clinical effect of FMT in SSc measured by the UCLA GIT score

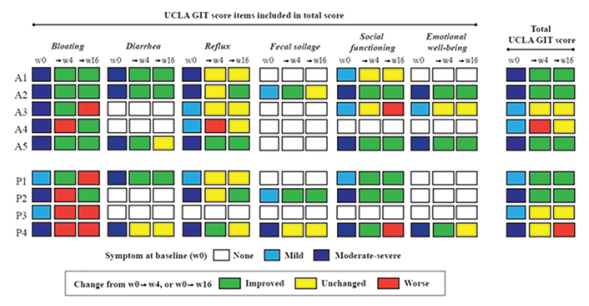

Stackplot of significantly changed relative abundance of fecal bacteria after FMT from week 0 to weeks 4 and 16

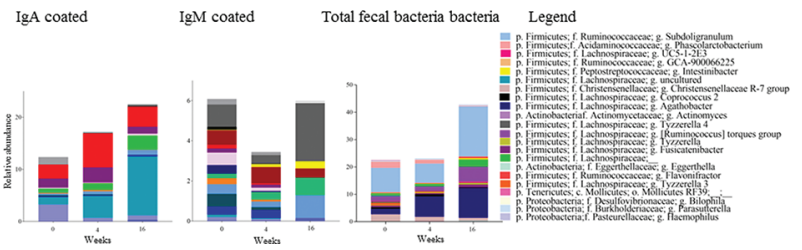

Conclusion: FMT of commercially-available ACHIM in patients with SSC appeared safe, effectively reduced lower GI symptoms, altered gut microbiota composition, richness and diversity and appeared to affect the mucosal immune system

Disclosure of Interests: Anna-Maria Hoffmann-Vold Grant/research support from: Received research funding or other remuneration from Boehringer Ingelheim, GSK, and Actelion, Consultant for: Received consulting fees or other remuneration from Boehringer Ingelheim, GSK, and Actelion, Speakers bureau: Actelion and Boehringer Ingelheim, Håvard Fretheim Consultant for: Received consulting fees or other remuneration from GSK, and Actelion, Brian K Chung: None declared, Henriette Didriksen Consultant for: Received consulting fees or other remuneration from GSK, and Actelion, Espen S Bækkevold: None declared, Øyvind Midtvedt: None declared, Cathrine Brunborg: None declared, Torhild Garen: None declared, Tore Midtvedt Shareholder of: Owner of ACHIM, Johannes R Hov: None declared, Knut EA Lundin: None declared, Øyvind Molberg: None declared

DOI: 10.1136/annrheumdis-2019-eular.4684

FRIDAY, 14 JUNE 2019

\section{Calming the cytokine storm in children and adults}

\begin{tabular}{|l|l}
\hline OP0328 & COMPARISON OF SERUM CYTOKINE PROFILE IN \\
MACROPHAGE ACTIVATION SYNDROME AMONG \\
DIFFERENT BACKGROUND RHEUMATIC DISEASES IN \\
CHILDREN:
\end{tabular}

Mao Mizuta, Masaki Shimizu, Masaaki Usami, Naoto Sakumura, Hitoshi Irabu, Maiko Takakuwa, Natsumi Inoue, Yasuo Nakagishi, Akihiro Yachie. Graduate School of Medical Sciences, Kanazawa University, Department of Pediatrics, Kanazawa, Japan

Background: Macrophage activation syndrome (MAS) is a severe, potentially life-threatening complication of pediatric rheumatic diseases. MAS occurs most often in children with systemic juvenile idiopathic arthritis (s-JIA) and less commonly in children with systemic lupus erythematosus (SLE), Kawasaki disease (KD) and juvenile dermatomyositis (JDM). The hallmark of MAS includes uncontrolled and dysfunctional immune responses involving continual activation and expansion of $\mathrm{T}$ lymphocytes and macrophages, which in turn lead to marked hypercytokinemia. However, it is still unknown which cytokines play a key role in the pathogenesis of MAS among different backgrounds.

Objectives: This study was aimed to clarify cytokines involved in the development of MAS among different background rheumatic diseases and to identify the serum biomarkers for the diagnosis of MAS.

Methods: Serum neopterin, interleukin (IL)-18, IL-6, tumor necrosis factor (TNF)$\alpha$ and soluble TNF receptor type I (sTNFR-I) and sTNFR-II levels were determined using enzyme-linked immunosorbent assay in 112 s-JIA patients including 30 with MAS, 8 SLE patients including 3 with MAS, 67 KD patients including 4 with MAS, and 7 JDM patients including 3 with MAS. Cytokine profiles in MAS phase of each disease were compared to those in active phase.

Results: Serum neopterin levels in patients with S-JIA, SLE and KD were significantly elevated in MAS phase compared to those in active phase. Serum neopterin levels in patients with JDM were also elevated in MAS phase compared to those in active phase, although statistically significant. Serum sTNFR-I levels in patients with S-JIA and SLE were significantly elevated in MAS phase compared to those in active phase. Serum sTNFR-I levels in patients with KD and JDM were also elevated in MAS phase compared to those in active phase, although statistically significant. Serum sTNFR-II levels in patients with s-JIA and KD were significantly elevated in MAS phase compared to those in active phase. Serum sTNFRII levels in patients with JDM were also elevated in MAS phase compared to those in active phase, although statistically significant. Serum IL-18 levels in patients with S-JIA were significantly elevated in both active and MAS phase compared to those in patients with other diseases. There were no significant differences of serum IL- 6 and TNF- $\alpha$ levels among different backgrounds.

Conclusion: The elevation of serum neopterin levels was the common finding in patients with MAS even in different backgrounds. These findings indicate that overproduction of interferon (IFN)- $\gamma$ might be closely related to the development of MAS. Serum neopterin levels which reflect IFN- $\gamma$ production might be a promising biomarker for the disease activity of MAS.

\section{REFERENCES:}

[1] Huber C, et al. J Exp Med 1984;160:310-6.

[2] Bracaglia C, et al. Ann Rheum Dis 2017;76:166-72.

Disclosure of Interests: None declared DOI: 10.1136/annrheumdis-2019-eular.6898

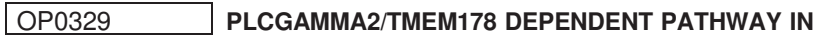 MYELOID CELLS MODULATES THE PATHOGENESIS OFCYTOKINE STORM SYNDROME}

Sahil Mahajan ${ }^{1}$, Zhengfeng Yang ${ }^{1}$, Elisabeth Mellins ${ }^{2}$, roberta faccio ${ }^{1,3 *}$.

${ }^{1}$ Washington University, Ortopaedics, St. Louis, United States of America;

${ }^{2}$ Stanford University, San Francisco, United States of America; ${ }^{3}$ Shriners Hospital for Children, St. Louis, United States of America

Background: Cytokine storm syndrome (CSS) is a life-threatening condition, observed in several rheumatic diseases, and a well-known complication of systemic juvenile idiopathic arthritis (SJIA) (1). Defects in T cell cytotoxicity leading to their excessive activation are major contributors to CSS development. However, dysregulated innate immune responses are also observed (2). The role of monocytes and macrophages as drivers of CSS, regardless of T cells, and the signaling pathways regulating monocyte/macrophage responses, have not been formally investigated.

Objectives: The goal of this study was to determine the contribution of monocytes/macrophages to the development of CSS and analyze the signaling pathways that lead to aberrant monocyte/macrophage pro-inflammatory responses using animal models of the disease and patient samples.

Methods: To study the involvement of monocytes/macrophages in a T celldependent model of CSS we treated LCMV-infected Perforin-/- mice with clodronate-liposomes or neutralizing anti-CSF1Ab prior to disease initiation. Similar depletion experiments were performed in a T-cell independent CSS model consisting of five injections of the TLR9 agonist CpG-1826 into WT mice. Myeloid cell contribution to CSS was assessed in mice with LysMCre-driven deletion of Plc $\gamma 2$ or global deletion of Tmem178, a downstream Plc 22 target gene specifically expressed in macrophages. Presence of immune infiltrates in liver and spleen was evaluated by FACS analysis and immunohistochemistry, circulating inflammatory cytokine levels were detected by ELISA, multi-organ damage was evaluated by histology.

Results: Depletion of monocytes/macrophages during early stages of CSS reduces mortality and inflammatory cytokine levels in both T-cell dependent and independent CSS models. Activation of Plc $\gamma 2$ in myeloid cells controls CSS development by driving macrophage pro-inflammatory responses. Intriguingly, the Plc $\gamma 2$ downstream effector Tmem178 acts in a negative feedback loop to restrain inflammatory cytokine production and protect from CSS development. Highlighting the clinical relevance, Tmem178 levels are reduced in macrophages from mice with CSS or exposed to SJIA patient plasma with active disease.

Conclusion: Macrophages are key drivers of CSS initiation. We identified a novel Plcy2/Tmem178 axis as a modulator of inflammatory cytokine production by monocytes/macrophages in CSS. Reduced Tmem178 levels are observed in SJIA and might be responsible for the increased macrophage pro-inflammatory responses.

\section{REFERENCES:}

[1] Canna SW, Behrens EM. Making sense of the cytokine storm: a conceptual framework for understanding, diagnosing, and treating hemophagocytic syndromes. Pediatr Clin North Am. 2012;59:329-44.

[2] Grom AA, Horne A, De Benedetti F. Macrophage activation syndrome in the era of biologic therapy. Nat Rev Rheumatol. 2016;12:259-68.

Acknowledgement: This work was supported by R01 AR053628 and R01 AR066551 from National Institute of Health (NIH) to Roberta Faccio and Shriners Hospital 85100 to Roberta Faccio. 